\title{
Study on water purification effects and microbial community compositions of the estuarine wetland buffer zones during a low-temperature period
}

\author{
Jiajun Sun ${ }^{1,2,4}$, Panyue Zhang ${ }^{3, *}$, Jinsong Liang ${ }^{3}$, Yajing Cai ${ }^{3}$, Yangyang Han ${ }^{5}$, Mengxiang \\ $\mathrm{Li}^{5}$, Zhi Wang ${ }^{6}$, and Yuping $\mathrm{Li}^{1}$ \\ ${ }^{1}$ Beijing Engineering Research Center of Process Pollution Control, Institute of Process Engineering, \\ Chinese Academy of Sciences, Beijing 100190, China \\ ${ }^{2}$ University of Chinese Academy of Sciences, Beijing 100049, China \\ ${ }^{3}$ College of Environmental Science and Engineering, Beijing Forestry University, Beijing 100083, \\ China \\ ${ }^{4}$ China Xiong'an Group Co. Ltd, Baoding071000, Hebei Province \\ ${ }^{5}$ College of Ecology and Environment, Hebei University, Hebei 071000,China \\ ${ }^{6}$ China Xiong'an Group Ecological Construction Investment Co. Ltd, No.2, Rongcheng071000, Hebei \\ Province
}

\begin{abstract}
The estuarine wetland buffer zone of the Fuhe river plays an important role in terms of improving water quality of Baiyangdian lake. In this study, the water purification effect and microbial community composition of an estuarine wetland buffer zone during a low-temperature period were investigated (March, 2021). The results indicated that the pre-sedimentation ecological pond (Pep), the subsurface flow wetland (Sfw), and the aquatic plant pond (App) exhibited good removal effects on total phosphorus (TP) and $\mathrm{NH}_{3}-\mathrm{N}$. In contrast, the removal effects on chemical oxygen demand (COD) and total nitrogen (TN) was unsatisfied. Various phosphorus removal bacteria were detected in the water samples from the Pep and the Sfw, such as Arthrobacter, Flavobacterium, and Planococcus. These findings indicated the water purification performance and the microbial community composition in an estuarine wetland buffer zone during a low-temperature period.
\end{abstract}

\section{Introduction}

Water environment and water ecology of an estuary directly affect the water quality and water ecological functions of lake and reservoir [1]. The construction of estuarine wetland buffer zones as ecological barrier can effectively intercept pollutant, protecting downstream water environment from pollution [2]. The aquatic plants, wetland structures, and microbial communities contribute to remove nitrogen and phosphorus in the estuarine wetland buffer zone [3].

\footnotetext{
*Corresponding author: panyue_zhang@bjfu.edu.cn
} 
The water purification performance of an estuarine wetland buffer zone is crucial to the water quality of Baiyangdian lake. The estuarine wetland buffer zone is located at the north of Jianchang village in Anxin county, which is the buffer zone of Fuhe river, Baohe river, and Caohe river, which drain into Zaozhadian Lake. The function of the estuarine wetland buffer zone is to purify water flowing into the Baiyangdian lake. Meanwhile, the design of the estuarine wetland buffer zone takes the emergency treatment of sudden water pollution accidents into account. The combined water treatment process of "pre-sedimentation ecological pond (Pep) + subsurface flow wetland (Sfw) + aquatic plant pond (App)" is applied in the estuarine wetland buffer zone. Chemical oxygen demand (COD), nitrogen, phosphorus, and other pollutants can be effectively removed by the combined water treatment process, the water quality of the effluent is up to the requirement of the national standards.

In this study, the water purification performance of the estuarine wetland buffer zone in Baiyangdian lake was investigated. According to the water quality monitoring data in March 2020 and the microbial community structure results, the water purification performance and the microbial community composition in the near natural wetland of the Fuhe river estuary during a low-temperature period were analyzed. This study provides a reference for the regulation of the natural wetlands.

\section{Materials and methods}

\subsection{Sampling}

Water samples were collected at the inlet and outlet of Pep, the outlets of Sfw, the submerged plant area, the emergent plant area, the Wanmu lotus-covered pond area, and the wetland. The water samples were collected below the water surface $0.5 \mathrm{~m}$ using the water sampler at 26-28 March 2021. The key water quality parameters were measured, including COD, total nitrogen (TN), total phosphorus (TP), and $\mathrm{NH}_{3}-\mathrm{N}$.

Eight sampling points were chosen for microbial analysis, in the Pep, the Sfw, and the App. Samples of water and sediment were taken from the Pep. The sampling points in the Sfw were set at the primary Sfw, the secondary Sfw, the three-stage Sfw zeolite layer, the three-stage Sfw gravel layer and the three-stage Sfw steel slag layer. The sampling points in the App were set at the submerged plant area, the Wanmu lotus-covered pond area and the emergent plant area. Sediment, fillers and water samples were collected from these different sampling points for microbial sequencing analysis.

\subsection{Analytical methods}

COD, TN, TP, and $\mathrm{NH}_{3}-\mathrm{N}$ were analyzed on the basis of the APHA methods [4]. All experiments were conducted in triplicate. High-throughput sequencing analysis was conducted at the platform of Majorbio Cloud Platform.

\subsection{DNA extraction and high-throughput sequencing analysis}

The DNA extraction was carried out using an E.Z.N.A. ${ }^{\circledR}$ soil DNA kit (Omega Bio-tek, Norcross, GA, U.S.) according to the manufacturer's protocols. The V3-V4 region 16S rRNA genes were expanded by employing 338F and 806R. Polymerase chain reactions (PCRs) were carried out as described in the study of Wang et al. [5]. The DNA library was built and loaded onto an Illumina MiSeq instrument and the amplicon library was completed on an Illumina MiSeq PE300 platform (Shanghai Majorbio Bio-pharm 
Techology Co., Ltd, China).

\section{Results and discussion}

\subsection{Effects of different treatment units and their contributions to water purification performance}

\subsubsection{Water purification performance of the pre-sedimentation ecological pond}

The COD, TN, TP, and $\mathrm{NH}_{3}-\mathrm{N}$ removal efficiencies of Pep were $-10.6 \%,-3.51 \%, 25.8 \%$, and $40.2 \%$, respectively (Table 1). Clearly, the removal efficiencies for neither organic pollutants nor TN were significant in the Pep, but better purification effects were found for $\mathrm{TP}$ and $\mathrm{NH}_{3}-\mathrm{N}$. Pep contributed to precipitate suspended solid particles and re-distribute water. Organic matter can be adsorbed on the surface of particles and removed with the settlement of particles. However, poor COD removal efficiency was related to the substrate releasement and strong hydraulic disturbance [6]. Poor TN removal efficiency was derived from the existence of bacteria, facilitating to convert ammonia nitrogen to nitrate nitrogen. Moreover, microbial decomposition of organic matter and its own metabolite processes might increase the TN concentration [7].

\subsubsection{Water purification performance of the subsurface flow wetland}

The COD, TN, TP, and $\mathrm{NH}_{3}-\mathrm{N}$ removal efficiencies of $\mathrm{Sfw}$ were $0 \%, 3.32 \%, 68.1 \%$, and $29.1 \%$ respectively (Table 1 ). Sfw exhibited good removal performances for TP and $\mathrm{NH}_{3}-\mathrm{N}$, rather than COD and TN. The nitrogen and phosphorus were removed from the water in Sfw by biodegradation and substrate adsorption. The TP removal efficiency was best in the Sfw, while the COD removal efficiency was the worst there. These effects might be caused by the low temperature in March, which could have inhibited the growth and metabolism of microorganisms and the potency of the aquatic plants, thus diminishing the COD removal efficiency [8].

\subsubsection{Water purification performance of the aquatic plant pond}

The COD, TN, TP, and $\mathrm{NH}_{3}-\mathrm{N}$ removal efficiencies of App were $11.5 \%, 4.04 \%, 66.7 \%$, and $48.7 \%$, respectively (Table 1). App exhibited good removal performances for TP and $\mathrm{NH}_{3}-\mathrm{N}$, rather than COD and TN, albeit higher than those in the Pep and Sfw. Oxygen could be generated by the submerged plant in App, which was used as the electron donor for heterotrophic bacteria and other autotrophic bacteria to degrade COD [9]. A soil layer with phosphorus removal capability was designed in the emergent plant area. The poor COD and TN removal efficiencies might be due to the release of nitrogen from sediments and the decrease of microbial growth and metabolism under low temperatures [10].

Table 1. Removal efficiencies of COD, TN, TP, and $\mathrm{NH}_{3}-\mathrm{N}$ in Pep, Sfw, and App.

\begin{tabular}{cccc}
\hline $\begin{array}{c}\text { Removal efficiency } \\
(\%)\end{array}$ & $\begin{array}{c}\text { Pre-sedimentation } \\
\text { ecological pond }\end{array}$ & $\begin{array}{c}\text { Subsurface flow } \\
\text { wetland }\end{array}$ & Aquatic plant pond \\
\hline Chemical oxygen & -10.6 & 0 & 11.5 \\
demand & & & \\
Total nitrogen & -3.51 & 3.32 & 4.04 \\
Total phosphorus & 25.8 & 68.1 & 66.7 \\
$\mathrm{NH}_{3}-\mathrm{N}$ & 40.2 & 29.1 & 48.7 \\
\hline
\end{tabular}




\subsection{Microbial analysis in different treatment units}

\subsubsection{Microorganism diversity and abundance analysis}

A total of 178475 high-quality effective sequences were detected, and 4144 OTUs (Operational Taxonomic Unit) were obtained with an average length of 420bp after $97 \%$ similarity analysis. Table 2 shows the diversity and abundance of microorganisms in the Pep, Sfw, and App. The Shannon and Chaol indexes represent the diversity and abundance of the microbial community, respectively [11].. The diversity and abundance of the sediment samples were significantly higher than those of the water and filler samples in the Pep, Sfw, and App. The results indicated that the microbial diversity in sediment was higher than that in water. The transformation and migration biochemical pathways were mainly contributed by the sediment microorganisms in the wetland ecosystem [12]. Meanwhile, Xu et al. [13] reported that the diversity and abundance of microorganisms in wetlands were affected by seasons, which were higher in autumn than in summer.

Table 2. The microorganism diversity and abundance in Pep, Sfw, and App.

\begin{tabular}{ccccc}
\hline Simple ID & Shannon & Simpson & Chao 1 & Coverage \\
\hline Pep_N & 6.713 & 0.0029 & 2837 & 0.9565 \\
Pep_S & 3.174 & 0.0691 & 392.4 & 0.9938 \\
Sfw_1 & 4.032 & 0.0576 & 1088 & 0.9816 \\
Sfw_2 & 3.537 & 0.1156 & 1074 & 0.9817 \\
Sfw_3 & 4.174 & 0.0941 & 1496 & 0.9876 \\
Sfw_4 & 3.805 & 0.0618 & 624.0 & 0.9832 \\
Sfw_5 & 3.661 & 0.1004 & 999.3 & 0.9906 \\
App_N_1 & 6.354 & 0.0052 & 2493 & 0.9622 \\
App_N_1 & 6.102 & 0.0105 & 2397 & 0.9627 \\
App_N_1 & 6.132 & 0.0065 & 2200 & 0.9671 \\
App_S & 4.033 & 0.0402 & 816.6 & 0.9863 \\
\hline
\end{tabular}

\subsubsection{Analysis of microbial community composition at phylum level}

Fig. 1 shows the microbial community composition in the sediment, water and filler samples at the phylum level. Total of 18 phyla were identified (at least 1\%). The dominant phyla being Proteobacteria, Actinobacteria, Firmicutes, Chloroflexi, Acidobacteria, Gemmatimonadetes, Cyanobacteria, and Nitrospirae. Li et al. [14] also found that Proteobacteria, Actinobacteria, Firmicutes were the dominant phyla in multi-level surface flow wetland matrix, among which Proteobacteria was the main.

The community structures in the sediment, water, and filler samples were significantly different at the phylum level (Fig. 1). The Proteobacteria reached $24.5 \%-56.2 \%$, $33.8 \%-68.0 \%$, and $15.5 \%-38.7 \%$ in the Pep, Sfw, and App, respectively. The Proteobacteria in the fillers and water samples was significant higher than that in the water samples. Proteobacteria play an important role in nitrogen and phosphorus removal in wetlands [15]. Therefore, large amounts of nitrogen and phosphorus were mainly removed in the water body and filler of different units. 


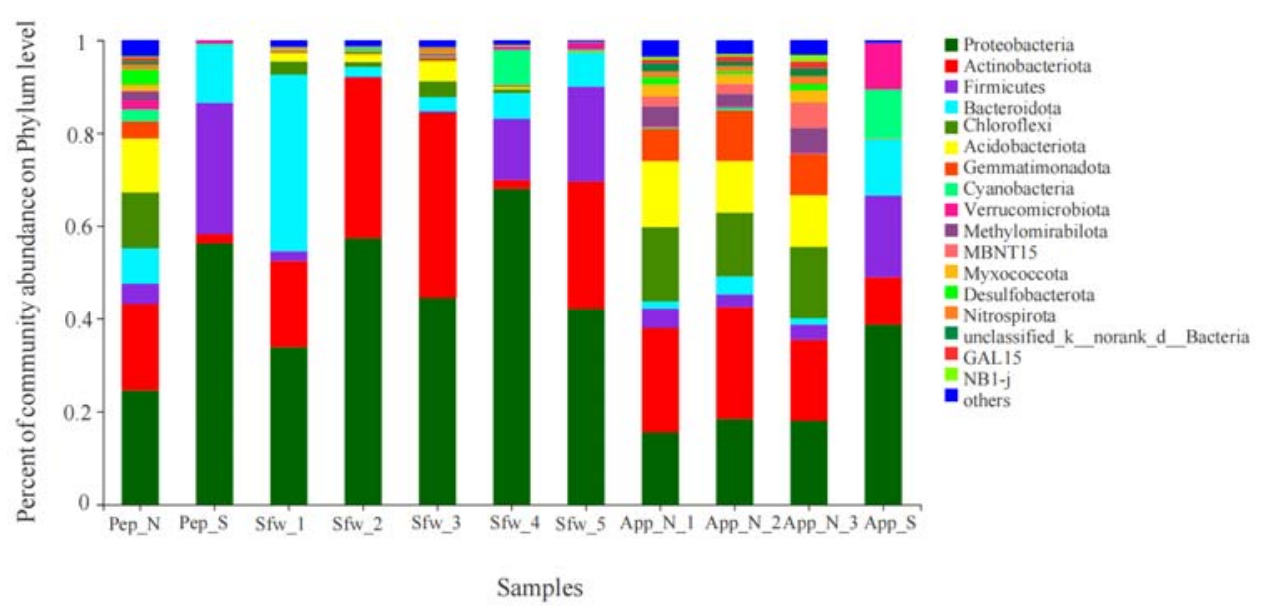

Fig. 1. Microbial community composition in sediment, water and filler samples at phylum level.

\subsubsection{Analysis of microbial community composition at genus level}

Fig. 2 shows the microbial community composition in sediment, water and filler samples at genus level. Total of 31 genera were identified. The dominant genera being Arthrobacter, Pseudomonas, Flavobacterium, Acinetobacter, Planococcus, Psychrobacter, Planomicrobium, Exiguobacterium, and some unclassified genera. Most of these are common wetland microorganisms, contributing to remove organic matter, nitrogen, and phosphorus [16]. As shown in Fig. 3, microbial community structures were different in the different treatment units, and the sediment samples exhibited significantly different microbial community structure characteristics from the water and filler samples.

The microbial community structures in the Pep showed significant differences between the sediment and water samples. The microorganisms in the sediment were Arthrobacter, Flavobacterium, Rhodoferax, and unclassified genera and bacteria, while the microorganisms in the water samples were Flavobacterium, Acinetobacter, Planococcus, Psychrobacter, and Planomicrobium. Flavobacterium and Acinetobacter, which could be classified to the heterotrophic denitrification bacteria, facilitating to remove phosphorus [17]. Planococcus and Planomicrobium played an important role in nitrate removal and cellulose degradation [18]. There many phosphorus-removing bacteria and denitrifying bacteria in water, but these bacteria are scarcer in sediment, and sometimes not even detected there. Therefore, the removal of $\mathrm{NH}_{3}-\mathrm{N}$ and TP is better in the Pep, but the effects on COD and TN are poor in the Pep (Table 1).

The microbial communities were significant differences in the different Sfw fillers samples (Fig. 2). The microorganisms in the filler samples were Arthrobacter, Pseudomonas, Flavobacterium, Acinetobacter, Planococcus, Psychrobacter, Nocardioides, and Planomicrobium. The Arthrobacter was $12.6 \%-28.6 \%$ in the Sfw fillers samples, which was significantly higher than that in the Pep and App samples. Arthrobacter widely exists in water and soil, facilitating to degrade aromatic compounds, nicotine, and 4-chlorophenol [19]. Compared to the Pep and App samples, many phosphorus removal microorganisms existed in the filler samples, especially in the three-stage Sfw steel slag layer sample. Therefore, Sfw plays an important role in the removal of organic matter, nitrogen, and phosphorus, especially phosphorus.

Similar to the results from the Pep, the microbial community structures in the App differed significantly between the sediment and water samples, with many unclassified genera and unknown bacteria. The Arthrobacter was $0.69-7.57 \%$ in the sediment samples, 
while the Flavobacterium, Planococcus, and Planomicrobium were lower in water than that of the Sfw samples owing to the absence of plants and the low temperature in March. The plants played an important role in App, and the interactions between plants and microorganisms promoted the removal of organic pollutants, nitrogen and phosphorus [20]. Therefore, the purification effect of the App will improve continually as the temperature increases and the plants grow.
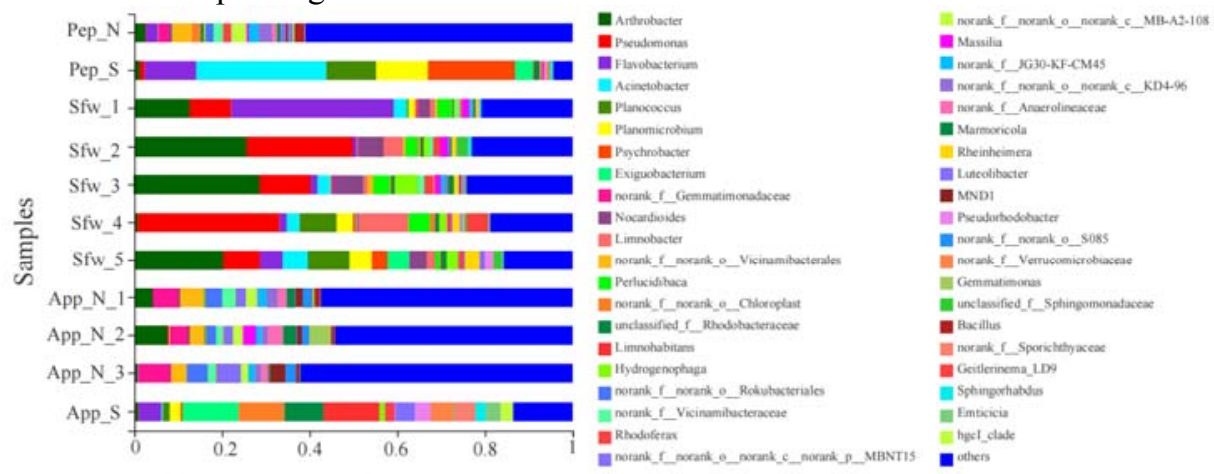

Percent of community abundance on Genus level

Fig. 2. Microbial community composition in the sediment, water, and filler samples at genus level.

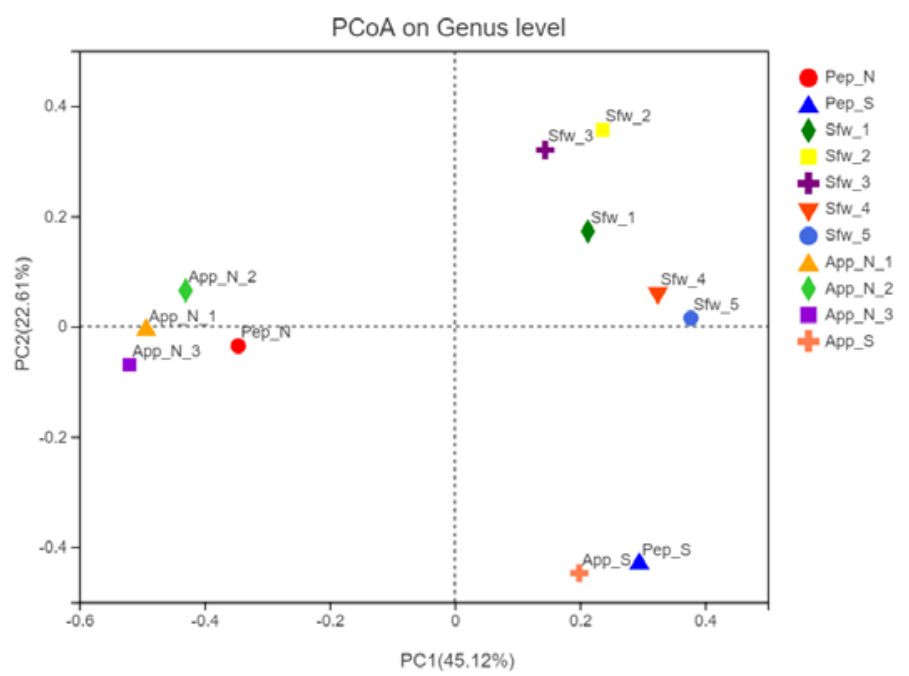

Fig. 3. PCoA analysis of the microbial community composition in the sediment, water, and filler samples.

\section{Conclusions}

The water purification effects and microbial community compositions of an estuarine wetland buffer zone were explored, during a low-temperature period (March). The TP and $\mathrm{NH}_{3}-\mathrm{N}$ removal efficiencies in each unit were better than those for TN and COD. The Pep, Sfw, and App experienced different water-quality treatment effects. The microbial diversity and community structures of the Pep, Sfw, and App showed significant differences. The Sfw harbored more nitrogen and phosphorus removal bacteria, which may have played an important role in water purification. Meanwhile, the low temperature and lack of flora might explain the poor water quality of the treatment units. This study will provide a 
reference for water purification effects and microbial community compositions of estuarine wetland buffer zones during low-temperature periods.

\section{Acknowledgements}

This work is supported by the National Water Pollution Control and Treatment Science and Technology Major Project (2018ZX07110003).

\section{References}

1. H. Zhang, W.Z. Tang, W.D. Wang, W. Yin, H.L. Liu, X.M. Ma, Y.Q. Z.H. Zhou, J.M. Zha. J. Environ. Sci. 104, 53-68. (2021)

2. Z.W. Ma, J.H. R. Xiao, C. Wang, Y. Cui, J. Wu, J. Xu, Z.M. Zhang, M.X. Zhang. Ecol. Indic. 121, 107309. (2021)

3. D. Parde, A. Patwa, A. Shukla, R. Vijay, R. Kumar. Environ. Technol. Inno. 21(4), 101261. (2020).

4. APHA. American Public Health Association, Washington, DC, USA. (2012)

5. S.Q. Wang, G.M. Zhang, P.Y. Zhang, X.W. Ma, F. Li, H.B. Zhang, X. Tao, J.P. Ye, M. Nabi. J. Environ. Manage. 220, 142-148. (2018)

6. W.A. Qian, Z.F. Cao, L. Qian, J.Y. Zhang, Y.B. Hu, J. Zhang, W. Xu, K. Qiang, X.C. Yuan, Q.F. Cheng. J. Environ. Manage. 249, 109398. (2019)

7. R. Aminitabrizi, R.M. Wilson, J.D. Fudyma, S.B. Hodgkins, M. M. Tfaily. Front. Earth Sci. 8, 557961. (2020)

8. J.B. Liang, Y. Yuan, Z.M. Zhang, S.J. You, Y.X. Yuan. Water, 13(9), 1152. (2021)

9. Y. Qiu, Y.L. Yu, H.N. Li, Z.Y. Yan, Z. Li, G.H. Liu, Z.H. Zhang, Y.J. Feng. Chem. Eng. J. 402, 125400. (2020)

10. M.N. Kumwimba, A. Batool, X.Y. Li. Sci. Total Envrion. 766, 142608. (2021)

11. L. Aljerf. Biodiversity Int. J. 1, 4-7. (2017)

12. Y.M. Shtarkman, Z.A. Kocer, E. Robyn, R.S. Veerapaneni, D. Tom, P. F. Morris. Plos One, 8(7), e67221. (2013)

13. K. Xu, R.Q.Wang, W.H. Guo, Z.D. Yu, R.L. Sun, J. Liu. Ecohydrol. Hydrobiol. 20(4), 570-583. (2020)

14. X. Li, Y. Li, Y. Li. Appl. Microbiol. Biotechnol. 102(24), 10755-10765. (2018)

15. S.Y. Bai, L.Q. Qin, L.H. Liu, X.T. Gao, Y.L. Ding, Y.H. Li. J. Environ. Manag. 280, 111682. (2020)

16. J. Xiao, J. Huang, M.J. Huang, M. Chen, M.Y. Wang. Bioresource Technol. 318, 124229. (2020)

17. X. Wang, H. Zhu, B. Yan, B. Shutes, H. Wen. Environ. Int. 138, 105628. (2020)

18. R. Zhuge, Q. Cheng, L. Mei, X. Song, L. Shi. BioResources. 15(1), 1732-1746. (2020)

19. L.L. Shi, P. Zhang, Y.H. He, F.Z. Zeng, J. Xu, L. He. Sci. Total. Environ. 771, 144831. (2021)

20. A. Raklami, A.I. Tahiri, N. Bechtaoui, E. Abdelhay, E. Pajuelo, M. Baslam, A. Meddich, K. Oufdou. J. Environ. Sci. 99, 210-221. (2021) 